\title{
Plasmonic LED device
}

\author{
Manuela Lunz ${ }^{a}$, Dick de Boer ${ }^{a}$, Gabriel Lozano ${ }^{b, c}$, Said R K Rodriguez ${ }^{b}$, Jaime Gomez Rivas ${ }^{\text {b, d }}$, \\ Marc A Verschuuren $^{\mathrm{a}}$ \\ ${ }^{\mathrm{a}}$ Philips Research, High Tech Campus 04, 5656AE Eindhoven, The Netherlands; ${ }^{\mathrm{b}}$ Center for \\ Nanophotonics, FOM Institute AMOLF c/o Philips Research, High Tech Campus 04, 5656AE

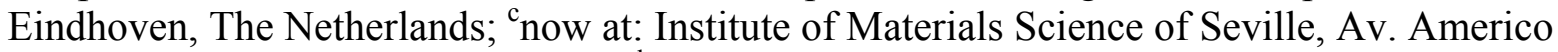 \\ Vespucio 49, 41092 Sevilla, Spain; ${ }^{\mathrm{d}}$ COBRA Research Institute, Eindhoven University of \\ Technology, Eindhoven, The Netherlands
}

\begin{abstract}
Plasmonic nanostructures are known to influence the emission of near-by emitters. They can enhance the absorption and modify the external quantum efficiency of the coupled system. To evaluate the possibility of using plasmonics to enhance the light emission of a phosphor-converted LED device and create an efficient directional light source, regular arrays of aluminium nanoparticles covered with a red dye layer are investigated. In arrays of aluminum nanocylinders with a diameter of ca $140 \mathrm{~nm}$ combined with a thin $(650 \mathrm{~nm})$ layer of luminescent material, very narrow resonances have been observed, which lead to large enhancement factors of up to 70 and 20 for excitation with a directional blue laser source and a lambertian LED respectively, in a small spectral range for particular angles. The measured resonances agree very well with finite-difference time-domain numerical simulations. These changes in the angular emission profile of the red dye as well as the spectral shape of its emission can help to optimize the efficacy of phosphor-converted LED modules and increase the amount of useable light in a certain angular cone. Using Fourier microscopy, large modifications of the angular emission profile as well as spectral shaping are observed for these plasmonic LED devices if compared to reference samples without plasmonic nanostructures.
\end{abstract}

Keywords: Plasmonics, surface plasmon lattice resonance, metal nanoparticles, phosphors, LED, spectral shaping, angular emission profile

\section{INTRODUCTION}

General lighting nowadays focuses almost completely on solid-state-based lighting systems, with white-light LED devices being available as retrofit bulbs but also in new form factors. White light can be generated by either combining direct emitting blue, green and red LEDs, conversion from near UV or blue to a broad white light spectrum via at least one phosphor or a combination of these two techniques.

An efficient light source is characterized by the three following aspects:

a) the generation of photons from electrical power with minimal losses,

b) a maximum of the generated photons illuminates the desired object, which can be for example a secondary optical element, or an open space,

c) the emission spectrum is optimized for the sensitivity of the detector, which in case of general lighting is the human eye.

A lot of progress has been made to improve the electrical LED efficiency mentioned above in a) since the first LED has been introduced. ${ }^{1,2}$ Furthermore, over the past few years a lot of attention has also been paid to point c), the optimization of the white light emission spectrum with respect to the human eye sensitivity curve. However, the latter has to be done with the boundary condition of still representing all the colors visible to the human eye, which is typically characterized by the color rendering index CRI. Several ways on how to achieve highly efficient systems while maintain an acceptable CRI have been proposed ${ }^{3}$ and realized ${ }^{4}$.

*manuela.lunz@philips.com

Photonic Crystal Materials and Devices XI, edited by Sergei G. Romanov, Gabriel Lozano,

Dario Gerace, Christelle Monat, Hernán Ruy Míguez, Proc. of SPIE Vol. 9127, 91270N

(C) 2014 SPIE · CCC code: 0277-786X/14/\$18 · doi: 10.1117/12.2052913

Proc. of SPIE Vol. 912791270 N-1 
It has been shown that periodic plasmonic metal nanoparticle (NP) arrays ${ }^{5}$ can largely enhance the photoluminescence of nearby emitters in particular directions for a certain narrow wavelength range. ${ }^{6-9}$ This enhancement corresponds, on the one hand, to a modification of the angular emission profile, as more light is emitted in a specific direction than in others at a particular wavelength. On the other hand it also corresponds to a modification of the spectrum in a particular direction or small solid angle, as in this angular range more emission occurs at certain wavelengths than at others. Therefore, using periodic plasmonic arrays in combination with LED phosphors can address both points b) and c) mentioned above simultaneously. Using such a plasmonic LED device could therefore show the following advantages over a regular phosphor-converted LED:

a) Modification of the angular emission profile such that the periodic plasmonic NP array could replace (partly) bulky secondary optical structures for focusing and collimating light in lighting applications.

b) Shaping of the emission to better fit the eye sensitivity, especially in the red part of the visible spectrum.

In this contribution, we evaluate the effect of a periodic NP array on a phosphor-converted LED device. We show that by using Fourier microscopy the angular emission profile of a plasmonic sample or LED device can be analysed for the full hemisphere of emission. Furthermore, we demonstrate that previously reported emission enhancements of 60 to 70 at very particular angles and wavelengths ${ }^{9}$ can translate into an enhanced emission in forward direction compared to a LED device with a regular phosphor layer only.

\section{EXPERIMENT}

The plasmonic samples investigated are periodic aluminum nanoparticle (NP) arrays fabricated by substrate-conformal imprint lithography and reactive ion etching on top of a glass substrate. ${ }^{10}$ The nanoparticles have a diameter of approximately $140 \mathrm{~nm}$ and a height of $150 \mathrm{~nm}$. The arrays were subsequently covered with a $650 \mathrm{~nm}$ thick dye layer, $3 \mathrm{wt} \%$ Lumogen F305 (BASF) in a polystyrene (PS) matrix, by spin coating. Dye references without plasmonic NP arrays were prepared directly on bare glass in the same way. The general structure of the plasmonic samples is shown in Figure 1(a). A scanning electron microscopy image of a square array with a pitch of $400 \mathrm{~nm}$ can be seen in Figure 1(b).
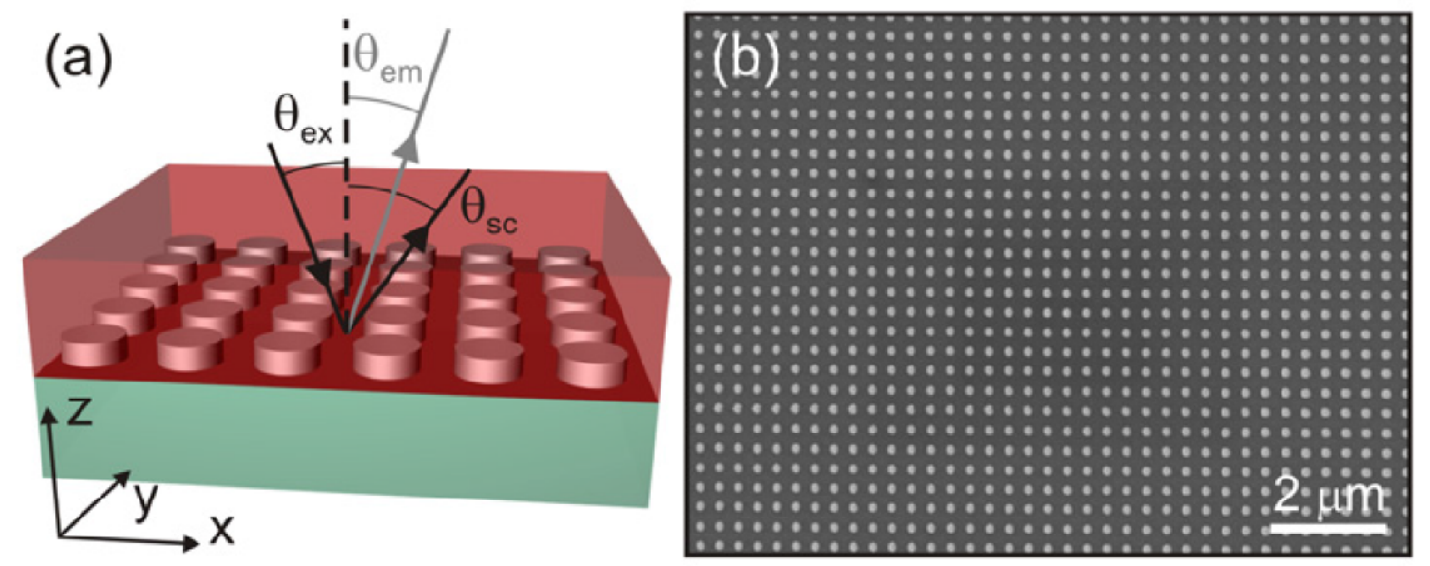

Figure 1. (a) Schematic of the sample structure indicating excitation angle $\theta_{\mathrm{ex}}$, angle of emission $\theta_{\mathrm{em}}$ and angle of scattered light $\theta_{\mathrm{sc}}$ with respect to the layer normal. ${ }^{14}$ The periodic square array is prepared on a glass substrate (green) and covered by a red dye layer (transparent red). (b) Scanning electron microscopy picture of the square aluminum nanoparticle array. ${ }^{14}$

The normalized absorption and photoluminescence emission spectra of a F305 dye layer on glass are shown in Figure 2. As can be seen from the photoluminescence spectrum, the dye emission shows a maximum in the wavelength range of $600-630 \mathrm{~nm}$ which is important to generate warm white light. However, there is also a significant amount of emission intensity detected for wavelengths longer than $650 \mathrm{~nm}$, wavelengths to which the eye is not very sensitive as indicated by the eye sensitivity curve also included in Figure 2. 


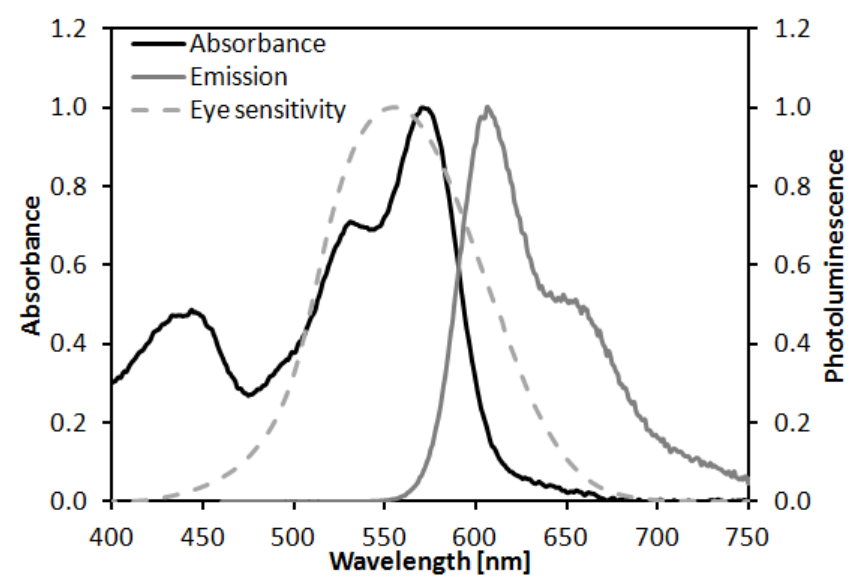

Figure 2. Normalized absorbance (black line) and photoluminescence (grey solid line) spectra of a red F305 dye layer on glass as well as the human eye sensitivity curve (grey dashed line).

To determine the spectral features of different plasmonic arrays, e.g. with nanoparticles of different sizes and different lattice constants, the angular dependent extinction as well as photoluminescence directional enhancement (PLDE) with respect to a dye-on-glass reference are evaluated under white light and blue laser excitation respectively. For a more detailed description of the technique see for example reference [9]. For a more in depth analysis of the emission profile, Fourier microscopy (FM) images of the samples under LED illumination were taken with narrow band pass filters. ${ }^{11-14}$ Compared to PLDE, where a line scan is taken at a fixed azimuthal angle $\phi$ to show the variation of the full emission spectrum with respect to the normal, FM shows the angular resolved emission intensity over the full hemisphere, however integrated over a narrow wavelength range per image.

\section{RESULTS AND DISCUSSION}

First of all, the samples were characterized by extinction and PLDE measurements, as shown in Figures 3(a) and (b) respectively. As can be seen, the narrow resonances in the PLDE measurement as function of the emission angle $\theta_{\mathrm{em}}$ correspond to the resonances seen in the extinction map in Figure 3(a) as function of the angle of light incidence $\theta_{\text {in }}$. These resonances follow the dispersion of Raleigh anomalies, indicated by the lines in Figures 3(a) and (b), and are known as surface lattice resonances (SLRs). ${ }^{5-7,9}$ For the current investigation, samples with emission enhancement at wavelengths in the range of ca $600-620 \mathrm{~nm}$ in forward direction were chosen. In Figure 3(b) a large enhancement of up to a factor 70 can be seen for p-polarized light in a wavelength range of ca 600 to $630 \mathrm{~nm}$ at emission angles $\theta_{\mathrm{em}}$ smaller than $5^{\circ}$.
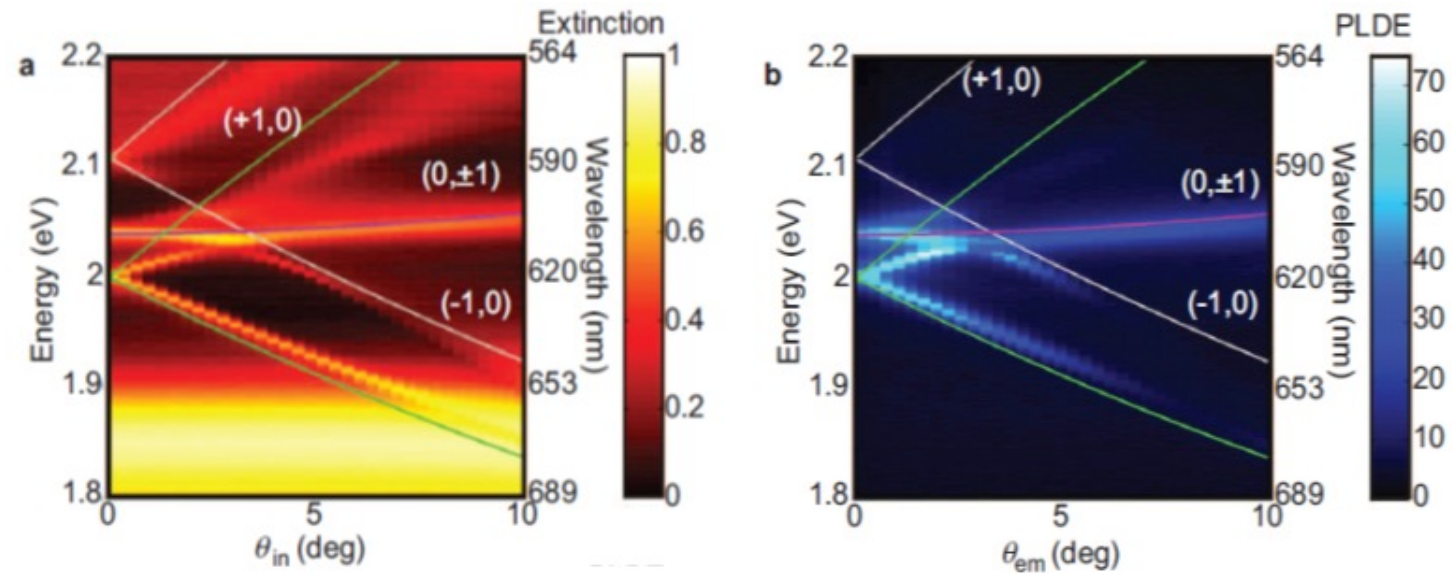

Figure 3. (a) Extinction map of p-polarized white light as function of energy / wavelength and angle of incidence $\theta_{\text {in. }}{ }^{9}$ (b) Ppolarized photoluminescence directional enhancement (PLDE) as function of energy / wavelength and emission angle $\theta_{\mathrm{em}}$ with excitation by a blue laser under normal incidence $\left(\theta_{\mathrm{ex}}=0^{\circ}\right){ }^{9}$ In both graphs the gray, purple and green lines indicate the Raleigh anomalies calculated for beams diffracted in a medium with refractive indices of $1.47,1.52$ and 1.55 , respectively. 
Numerical simulations by 3D finite-difference time-domain calculations were carried out and showed very good agreement with the measured PLDE. ${ }^{9}$ The simulations furthermore confirm that the measured enhancement is due to two effects, an enhancement of the excitation of the phosphor by the pump light as well as enhanced out-coupling of the emission in certain directions.

As mentioned above, the PLDE is measured at a single azimuth angle $\phi$, while the intensity at a particular emission angle $\theta_{\mathrm{em}}$ will change as a function of $\phi$ for a plasmonic sample. In order to determine how the 60 to 70 fold enhancement of the photoluminescence translates in an application, the full hemisphere of emission has to be analyzed and compared to the reference. Therefore, a dye covered square NP array and dye reference illuminated with a blue LED were investigated by Fourier microscopy (FM) for the wavelength range of interest with a band-pass filter centered at $600 \mathrm{~nm}$ $( \pm 5 \mathrm{~nm}, \mathrm{FWHM})$. The samples are very similar to the ones discussed above; the only difference is that the samples for LED illumination extend over a larger area in order to cover the complete LED device.
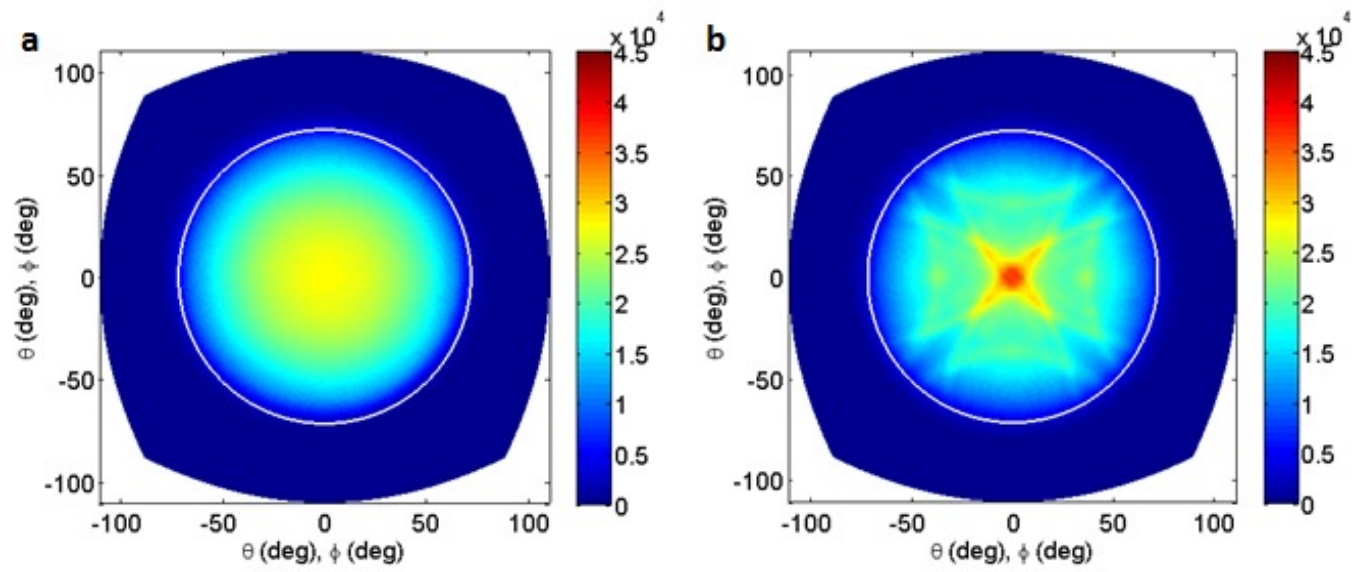

Figure 4. Fourier images of the emission (color scale in counts/s) as a function of emission angle $\theta$ with respect to the sample normal and azimuthal emission angle $\phi$ for a LED device with a (a) red dye layer on glass and (b) a red dye layer covering a regular square array of aluminum nanoparticles (pitch $400 \mathrm{~nm}$ ). The white circles indicate the maximum angle of $72^{\circ}$ detected, limited by the NA of the microscope objective.

The FM image of the reference can be seen in Figure 4(a) and shows a rather homogeneous and smooth profile close to Lambertian. In contrast, the FM image of the dye layer on top of the square NP array, presented as Figure 4(b), shows distinct angular features with a four-fold symmetry typical for a square NP array. ${ }^{14}$ As can be seen, measuring the intensity profile along the line of highest emission (diagonals in Figure 4(b)) will give a different absolute intensity level than for an azimuthal angle $\phi$ shifted by $45^{\circ}$ (horizontal or vertical cross section). Therefore, it is very important to take into account the intensity distribution over the whole hemisphere when evaluating the application potential of plasmonic structures.

Furthermore, it can be clearly seen that in the center of the image, corresponding to an emission normal to the sample surface, i.e. in forward direction, a higher intensity is detected for the plasmonic sample (Figure 4(b)) than for the dye reference (Figure 4(a)). To evaluate this further and to determine the difference in overall emission in a particular cone with angle $\theta$, the intensity detected in the FM images is integrated radially, i.e. over the azimuthal angle $\phi$, as well as from the center up to a particular value of the emission angle $\theta$ with respect to the layer normal. The ratio of the integrated intensity of the plasmonic sample with respect to the reference sample is shown as a function of this angle $\theta$ (the maximum angle of the cone) in Figure 5. When integrating over a small cone of $5^{\circ}$, an emission enhancement by a factor of 1.3 can be achieved for the wavelength band at $600 \mathrm{~nm}$. When increasing the cone, the enhancement decreases as the plasmonic enhancement decreases and the resonances shift to different wavelengths, as can be seen in the PLDE map in Figure 3(b) as well as from the FM image in Figure 4(b). For angles larger than $20^{\circ}$, the ratio of integrated intensity even drops to below 1, indicating that there are losses within our plasmonic system due to absorption by the metal NPs. The large difference between the 1.3 enhancement measured with the Fourier microscope and the 60 to 70 enhancement reported in Fig.3 (a) is due to several factors. First, the illumination in the last measurement is done with the collimated beam of a laser incident at a resonant angle, which enhances the absorption by the phosphor. Second, the Fourier microscope measurements are done with a band pass filter with a bandwidth of $10 \mathrm{~nm}$, while the maximum enhancement is achieved in a narrower range of wavelengths. Finally, the 1.3 times enhancement is the integrated value 
over a range of angles spanning from $-5^{\circ}$ to $5^{\circ}$, while the maximum enhancement is limited to a well defined angle (see Fig. 3(b)).

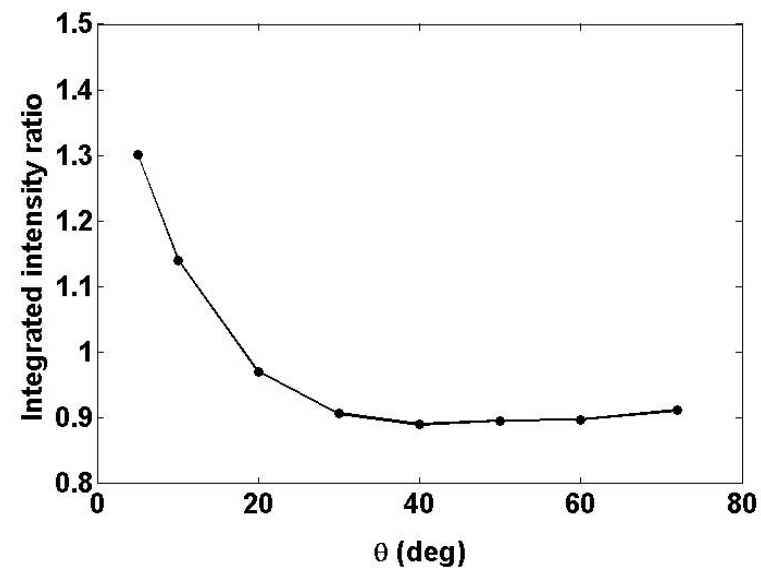

Figure 5. Relative emission of a plasmonic LED device with a dye layer on top of a regular square NP array with respect to a pure dye layer on glass as a function of azimuthal emission angle $\theta$. The relative intensity was calculated by integrating the Fourier images shown in Figure 4 over the azimuthal angle $\phi$ and up to the elevation $\theta$.

Having established a way in which the angular emission profiles of plasmonic samples can be evaluated with respect to reference phosphor layers, arrays with different pitches and geometries can now be evaluated with respect to their suitability to tune the LED emission spatially and spectrally. It has however to be kept in mind that this is a relative measurement and in order to really quantify the absolute efficacy of a plasmonic LED device a calibrated measurement setup is required.

\section{CONCLUSION}

Altogether, we have shown that large emission enhancements of up to a factor of 70 can be observed for emitters interacting with periodic plasmonic nanoparticle arrays supporting surface plasmon lattice resonances at specific angles and wavelengths. These features can be used to tune the angular intensity profile as well as the emission spectrum of phosphors used for white light generation in LED devices. Using Fourier microscopy, it has been shown that, within a narrow band of wavelengths, the emission of a plasmonic LED device is enhanced in a narrow cone of $5^{\circ}$ with respect to the reference dye layer without plasmonic NPs. In a next step different nanoparticle shapes, array pitches and geometries will be investigated to find the optimum nanoparticle lattices that can be compared in this way to optimize the performance of a plasmonic LED device.

\section{ACKNOWLEDGEMENTS}

We thank Remco van Brakel for his support with the sample preparation as well as Grzegorz Grzela and Mohammad Ramezani for their help with measurements and data analysis. This work is partially financed by the Foundation for Fundamental Research on Matter (FOM), which is financially supported by the Netherlands Organization for Fundamental Research (NWO). It is also part of an industrial partnership program between Philips and FOM. It is also supported in part by NanoNextNL, a micro and nanotechnology consortium of the Government of the Netherlands and 130 partners. 


\section{REFERENCES}

[1] Zheludev, N. "The life and times of the LED - a 100-year history", Nature Photonics 1, 189 - 192 (2007).

[2] Pimputkar, S., Speck, J. S., DenBaars, S. P., and Nakamura, S., "Prospects for LED lighting", Nature Photonics 3, 180 - 182 (2009).

[3] Phillips, J. M., Coltrin, M. E., Crawford, M. H., Fischer, A. J., Krames, M. R., Mueller-Mach, R., Mueller, G. O., Ohno, Y., Rohwer, L. E. S., Simmons, J. A., and Tsao, J. Y., "Research challenges to ultra-efficient inorganic solid-state lighting", Laser \& Photon. Rev. 1 (4), 307 - 333 (2007).

[4] "Details of the $200 \mathrm{~lm} / \mathrm{W}$ TLED lighting technology breakthrough unraveled", 11 April 2013, http://www.newscenter.philips.com/main/standard/news/articles/20130411-details-of-the-200lm-w-tledlighting-technology-breakthrough-unraveled.wpd\#.UyBoQoUd2rk .

[5] Auguie, B., and Barnes, W. L., "Collective Resonances in Gold Nanoparticle Arrays", Phys. Rev. Lett. 101, 143902 (2008).

[6] Vecchi, G., Giannini, V., and Gómez Rivas, J., "Shaping the Fluorescent Emission by Lattice Resonances in Plasmonic Crystals of Nanoantennas", Phys. Rev. Lett. 102, 146807 (2009).

[7] Rodriguez, S. R. K., Lozano, G., Verschuuren, M. A., Gomes, R., Lambert, K., De Geyter, B., Hassinen, A., Van Thourhout, D., Hens, Z., and Gómez Rivas, J., "Quantum rod emission coupled to plasmonic lattice resonances: A collective directional source of polarized light", Appl. Phys. Lett. 100, 111103 (2012).

[8] Rodriguez, S. R. K., Murai, S., Verschuuren, M. A., and Gómez Rivas, J., "Light-Emitting Waveguide-Plasmon Polaritons", Phys. Rev. Lett. 109, 166803 (2012).

[9] Lozano, G., Louwers, D. J., Rodriguez, S. R. K., Murai, S., Jansen, O. T. A., Verschuuren, M. A., and Gómez Rivas, J., "Plasmonics for solid-state lighting: enhanced excitation and directional emission of highly efficient light sources", Light: Sci. Appl. 2, e66 (2013).

[10] Verschuuren M. A., "Substrate Conformal Imprint Lithographhy for Nanophotonics", PhD thesis, Utrecht University, Utrecht, The Netherlands, 2010.

[11] Yoshita, M., Koyama, K., Baba, M., and Akiyama, H., "Fourier imaging study of efficient near-field optical coupling in solid inmersion fluorescence microscopy", J. Appl. Phys. 92, 862 (2002).

[12] Gómez Rivas, J., Vecchi, G. and Giannini, V., "Surface plasmon polariton-mediated enhancement of the emission of dye molecules on metallic gratings", New J. Phys. 10, 105007 (2008).

[13] Grzela, G., Paniagua-Domínguez, R., Barten, T., Fontana, Y., Sánchez-Gil, J. A., and Gómez Rivas, J., "Nanowire Antenna Emission", Nano Lett. 12, 5481-5486 (2012).

[14] Lozano, G., Barten, T., Grzela, G., and Gómez Rivas, J., "Directional absorption by phased arrays of plasmonic nanoantennae probed with time-reversed Fourier microscopy", New J. Phys. 16, 013040 (2014). 